\title{
Acquisition of Plate-making Process Knowledge for Smart Clothing CAD Systems *
}

\author{
Huijie Guo, Boan Ying*, Xin Zhang, Jing Qi \\ Apparel \& Art Design College, Xi'an Polytechnic University, Xi'an, Shaanxi 710048, China
}

\begin{abstract}
The digital design of the clothing model currently existing in the clothing enterprise is still only an auxiliary tool in the sense of drawing. This type of garment computer-aided design (CAD) systems can not integrate the design principles and knowledge of the clothing professional field, similar design and expert experience into the system, which leads to the need for the plate-making to rely on the personal experience of the pattern maker, and there is a problem of low design efficiency. The intelligent clothing CAD system for tailoring the needs of clothing personalization can solve the defects of traditional CAD by curing the knowledge of clothing layout design. In this paper, through the method of plate-making experiment, the knowledge of garment plate-making process is analyzed and refined, and the garment model design model is constructed by using directed graph. The method of applying the model to smart garment CAD systems is illustrated by an example.
\end{abstract}

Keywords: Intelligent Plate-making; Knowledge Acquisition; Directed Graph

\section{Introduction}

Although the clothing auxiliary design systems in the traditional sense has been widely used in garment manufacturing enterprises, such systems cannot fully utilize the knowledge in the field, resulting in the inability to realize the reuse of knowledge resources and integration with other design links. Designers still need to a lot of repetitive design work has been carried out, which seriously affects the efficiency of the design of the garment model and the innovative work of the designer. Therefore, people in the clothing profession have conducted different levels of research on knowledge-driven CAD systems, first of all in the field of parametric plate-making technology. Hong Lu [1] used the turning collar as the research object to derive the relationship formula between the lodging amount and the lapel looseness and its changing law, which laid a theoretical foundation for the parametric design of the turning collar. Jie Feng [2] analysed key factors such as roll basic point and collar roll ease and realized the parametric design of a

${ }^{\star}$ Project supported by the China Textile Industry Federation and Textile Light Technology Education Foundation,Research on Constructing a Garment Pattern Design Model for Garment Intelligent Design (Project No. J201606).

*Corresponding author.

Email address: yingba2006@163.com (Boan Ying). 
rolled collar pattern based on clothing CAD. Yuan-yuan Zhang [3] discussed the principle and the latest technology of digital manufacturing of garment enterprises and obtained digital platemaking techniques for men's suit collars. Li-jun Jiang [4] proposed a three-dimensional model for a collar structure based on the mathematical model of personalized neck features. Feng Feng [5] expounded the design principle of a dynamic garment structure through the conversion of apparel structure diagrams into "directed graphs".

The level of achievement concerning the intelligent clothing plate-making system depends on the accumulation of professional knowledge in the field. Many scholars have also conducted different levels of research on the acquisition of professional apparel knowledge. Chang-peng $\mathrm{Hu}$ [6], based on the analysis of suit pattern expert knowledge, used parametric and modular design methods to achieve the intelligent production of the pattern of the suit. Ze-hong Jiang [7], based on customization production, used the method of plate-making experimentation to record the knowledge of men's suit plate-making processes and establish a parameterized relationship model to improve the efficiency of plate-making. Mei-mei Zhao [8] obtained and expressed the knowledge in the process of uniform design based on case design theory and established a knowledge management system, as well as an example database that can be used to calculate and retrieve similar design results. Jin Duan [9], based on the needs of personalized clothing, established a menswear type relationship model through the idea of layered design in order to provide a scientific basis for the establishment of knowledge-driven personalized sizes of male body type in the automatic design and generation of models. Wen-can Zhou [10] constructed clothing field ontology for clothing plate-making knowledge and realized the automatic plate-making of the suit semantic pattern described by the plate maker, which got rid of the dependence on the sample library and plate-making tools and improved the plate-making efficiency.

The existing research results have not been based on the development of intelligent software systems to acquire the process knowledge of garment plate-making. Therefore, this paper proposes a method of knowledge acquisition based on the process of men's suit collar plate-making. This method aims to achieve the acquisition of explicit and implicit knowledge in the process of platemaking and hopes to lay a theoretical foundation for the automation of garment knowledge-based work software.

\section{Plate-making Process Knowledge}

Software is a tool for knowledge manifestation. The amount of knowledge determines the level of manufacturing and the level of knowledge solidification determines the efficiency of software design. The acquisition and representation of the clothing type design knowledge is the core of the intelligent clothing CAD system that is adapted to the tailor-made production mode of the clothing. Therefore, it is necessary to analyze and summarize the knowledge of the garment plate-making process.

The pattern is a special language of the modern clothing industry, which is the symbol and process parameter basis for the garment design to enter the substantive stage [11]. The knowledge of the garment plate-making process studied in this paper is the process knowledge of pattern design using the design pattern of the prototype pattern and the method of plane drawing. That is, the procedural knowledge of garment plate-making is a description of how to manipulate anthropometric data to achieve the result of garment pattern drawing. 


\subsection{Explicit Design Knowledge}

Explicit knowledge has certain identifiability. In the process of garment making using the prototype method, the concepts, rules, patterns or rules used in the pattern drawing are all explicit design knowledge.

Take the male suit plate-making process as an example. The length of the coat, the bust, the amount of money, and the height of the sleeves used in the pattern drawing process are conceptual knowledge. The bust calculation formula $\mathrm{B} / 2+10$ and the back collar width formula B/12 used in the drawing of the prototype of the male suit are the knowledge of the plate-making law, that is, the body measurement size corresponds to the design size of the male suit. The male suit style elements mainly include the front piece, the back piece, the sleeve and the collar. The platemaking steps are also designed according to the pattern knowledge. To classify and summarize the constraint relationship between the dotted lines in the plate-making process, you can get the rule knowledge, such as the point-finding method (coordinate point, inner bisector, vertical intersection, etc.) and the dotted line connection method (connecting line segments, drawing parallel lines, draw free curves, etc.).

\subsection{Implicit Design Knowledge}

Tacit knowledge is knowledge that exists in the individual's mind, in a certain environment, is difficult to formalize and difficult to communicate, and is a key part of knowledge innovation. The design part of the knowledge of the garment plate-making process often exists in the brain of the plate maker in the form of experience, technology and know-how, which is relatively difficult to obtain.

Take the process of making a man's suit as an example. There are many factors affecting the shape of the collar, including the serial line position, the width of the head and the shape of the collar. Considering the comfort of the human neck, the height of the collar of the tumbling collar is generally $2.5 \sim 5 \mathrm{~cm}$, while the width of the lap collar is determined by the shape, and the minimum can cover the collar. In addition to the width of the lapel, the collar angle can be pointed or upturned or drooped. The height of the collar is limited by the comfort of the neck movement, but the width of the collar is large enough to cover the lower limit of the collar, and can be widened to cover the entire shoulder. When the height of the collar is high, the width of the collar is larger, and the difference between the upper and lower lines of the collar is larger. The shape of the collar is also inclined, and the curvature of the collar is larger. The analysis of the various positions of the collar type, the value of the experience value requires the people who have the experience of the plate to summarize many samples.

\subsection{Plate-making Process Knowledge Acquisition}

In this paper, the male suit is used as the research object, and the plate-making process is used to obtain the knowledge of the plate-making process. It aims to record the explicit knowledge and implicit knowledge contained in the process of the collar structure as far as possible from the thinking of the cartographer in order to improve the reuse of the design results and lay the foundation for realizing knowledge automation.

This experiment uses the V2017 version of the ET-CAD system from Cloth Easy Technology, 
according to the set style parameters of the flat collar style, shown in Table 1 . In addition, the serial cable line of the flat collar style is set to be tangent to the curve of the front collar, and the intersection of the door line and the waistline is used as a barge point, as shown in Fig. 1. The serial cable is tangential to the front collar arc, while the intersection of the front centre line and the waist line is the point of affiliation. Among them, the values of $a$ and $b$ and the position of the point of affiliation are the criteria for the suit lapel [12].

Table 1: Flat collar style parameters

\begin{tabular}{ccccccc}
\hline $\begin{array}{c}\text { Style } \\
\text { parameters }\end{array}$ & Collar(a) & $\begin{array}{c}\text { collar } \\
\text { stand(b) }\end{array}$ & $\begin{array}{c}\text { rolled collar } \\
\text { width(c) }\end{array}$ & $\begin{array}{c}\text { lapel collar } \\
\text { width }(\mathrm{d})\end{array}$ & $\begin{array}{c}\text { lapel } \\
\text { collar }(\mathrm{e})\end{array}$ & $\begin{array}{c}\text { collar angle } \\
\text { equal }(\alpha)\end{array}$ \\
\hline Numerical value & $3.5 \mathrm{~cm}$ & $2.5 \mathrm{~cm}$ & $3.5 \mathrm{~cm}$ & $4 \mathrm{~cm}$ & $8.5 \mathrm{~cm}$ & $90^{\circ}$ \\
\hline
\end{tabular}

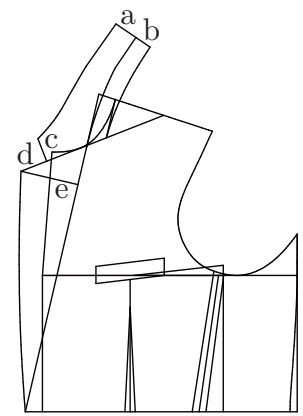

Fig. 1: Experimental collar style parameters

According to the above style and style parameters setting, the male suit flat collar is drawn, as shown in Fig. 2. Among them, the key plate-making statements of the fold line and the lapel part are shown in Table 2.

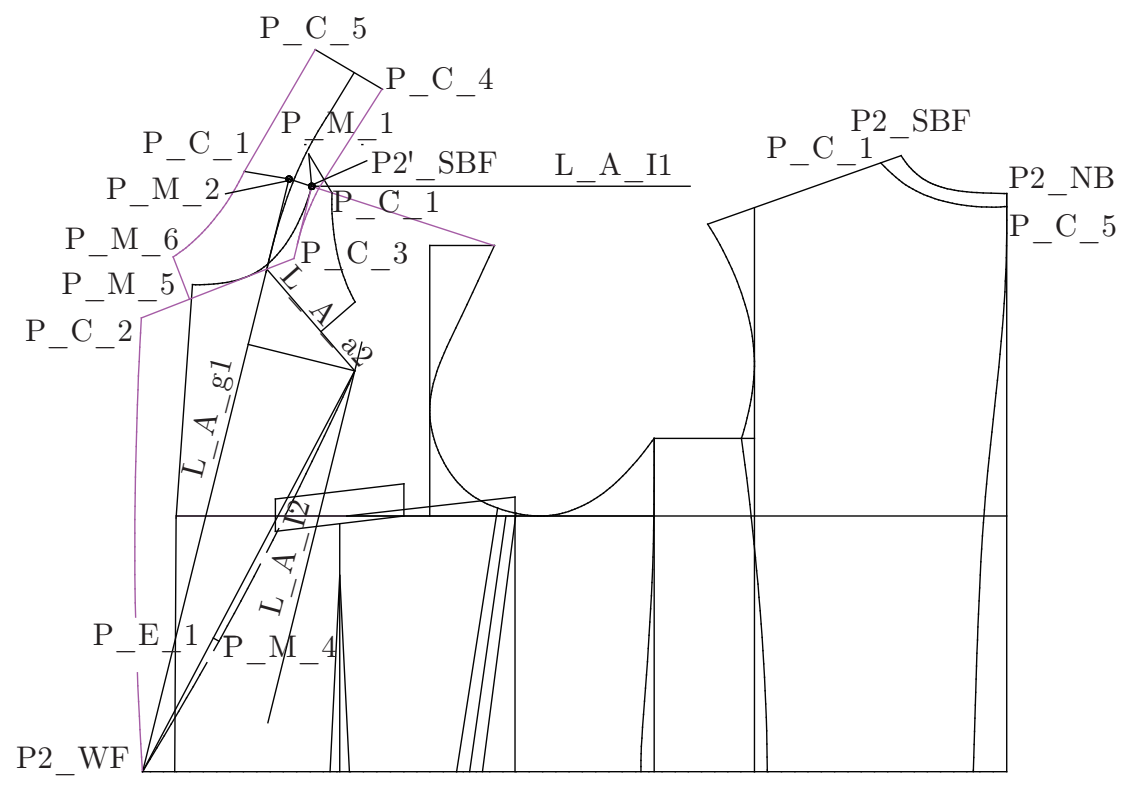

Fig. 2: Plate-making results of men's suits collar 
Table 2: Description of suit collar key plate-making statement

\begin{tabular}{|c|c|}
\hline $\begin{array}{c}\text { Plate-making } \\
\text { part }\end{array}$ & Description statement \\
\hline Fold line & 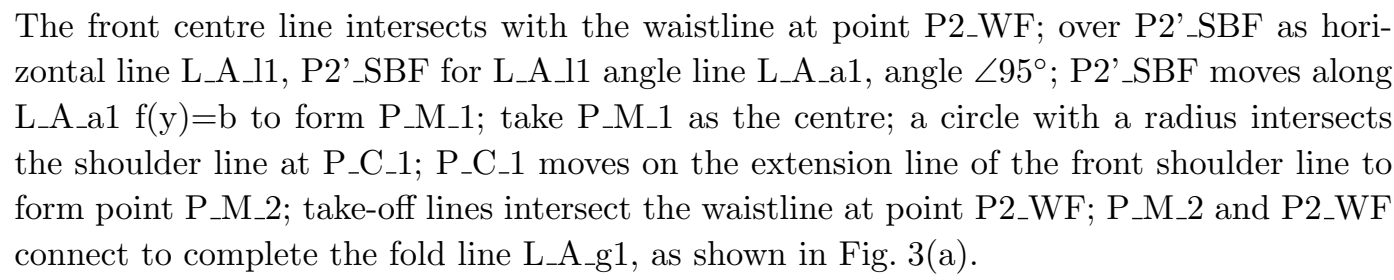 \\
\hline $\begin{array}{l}\text { Rolled collar } \\
\text { structure }\end{array}$ & 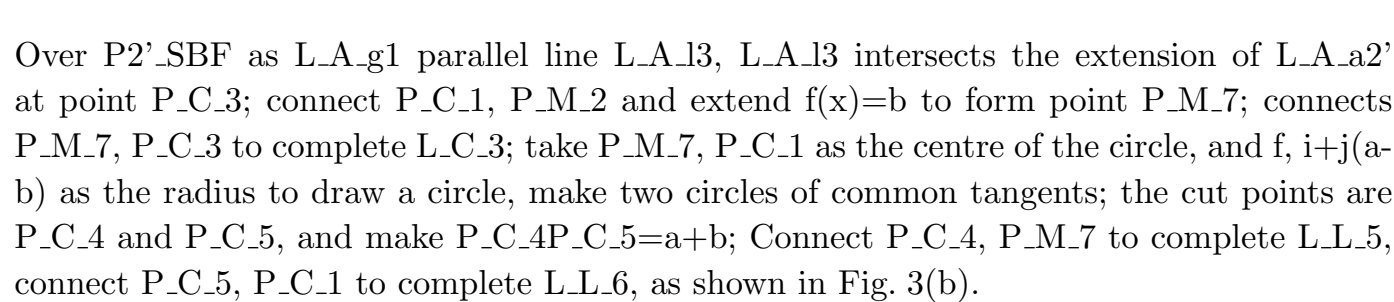 \\
\hline
\end{tabular}

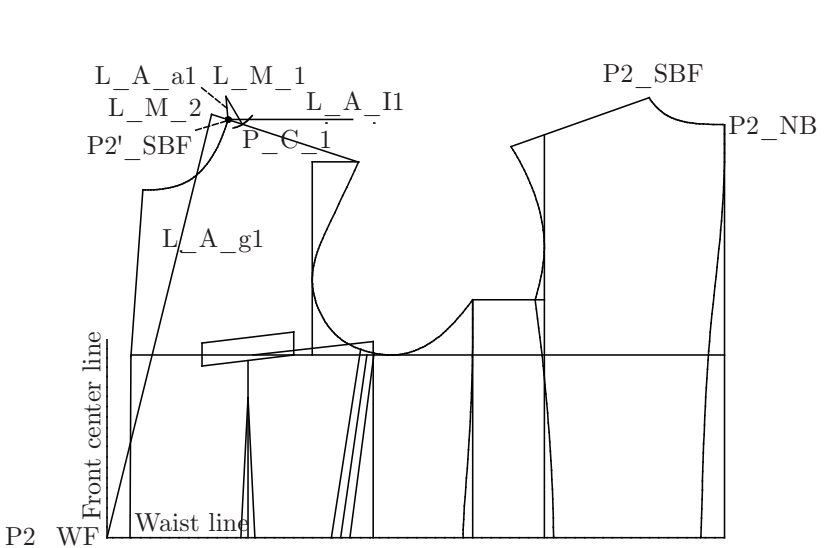

(a) Fold line

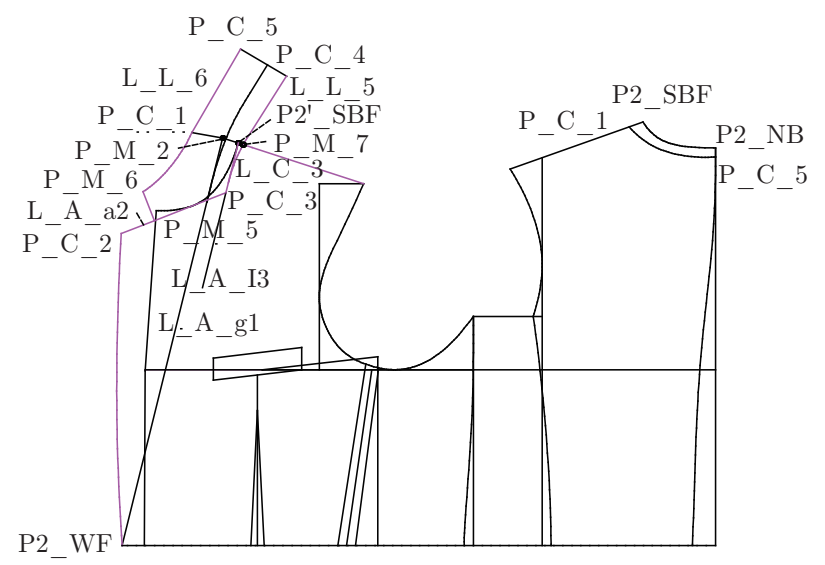

(b) Rolled collar structure

Fig. 3: The result of the plate-making to the description statement

According to the above experimental results, the acquisition of traditional clothing plate-making knowledge is recorded in the form of a structure diagram, but this form does not fully express the empirical knowledge of the cartographer implied in the diagram, resulting in poor readability and reproducibility of clothing structure diagrams. This plate-making experiment specifically records the process of plate-making in tabular form for the drawbacks of the traditional plate-making method. In this way, the empirical knowledge of the point, line generation method, calculation formula, rules and plate-making order used in the plate-making process is clearly expressed.

\section{Plate-making Process Knowledge Model}

The method of recording the knowledge of the plate-making process in words makes it easier to understand the cartographer's intention than the plane structure chart; but, because of the complicated and intertwined process, it is difficult to modify the plate-making process. The 
intelligent plate-making system not only requires the convenience of local structure modification, but also requires the calculation and processing efficiency of the plate-making process. Therefore, this paper uses the directed graph model to intuitively and clearly express the knowledge of the plate-making process, aiming to refine the explicit and implicit knowledge and design rules in the design process, thereby realizing the automation of design knowledge. According to a parallel program based on the weighted set of serial programs proposed by Zhang Peng [13] and 2.3 experimental contents, the knowledge of the plate-making process is expressed in the form of directed graphs, and the model is analyzed. The aim is to refine the explicit and implicit knowledge and design rules in the design process, so as to realize the automation of design knowledge.

\subsection{Model Construction Theory}

There is a vertex set $V=\left\{v_{1}, v_{2}, \cdots, v_{n}\right\}$ and an edge set $E=\left\{e_{1}, e_{2}, \cdots, e_{m}\right\}$, if for any edge $e_{k}$ in $E$, there is a vertex pair $\left(v_{i}, v_{j}\right)$ in $\mathrm{V}$ corresponding to it, we call the group consisting of $\mathrm{V}$ and $\mathrm{E}$ a graph, denoted as $G=(V, E)$. When two vertices associated with an edge are ordered, such a graph is called a directed graph [14].

This paper uses the basic theory of directed graphs to construct a knowledge directed graph model for garment plate-making process. The garment plate-making process knowledge directed graph is a graphical representation of the garment layout drawing process. The geometry is constructed by sequentially recording the history of a geometry's construction method. The method focuses on the process and method of generating the results.

The plate-making process is to first determine a point, and then determine another point based on the clothing knowledge (plate-making formula, size formula, empirical data, etc.) and then determine more points. The two points are connected into a line or a plurality of points into a curve or a face, and the process is repeated until a piece of clothing is formed. Therefore, the garment plate-making process knowledge directed graph model describes all the points, line elements and their mutual relations that appear in the plate-making process as a directed graph structure. The point and line elements correspond to the vertices of the directed graph, and the relationship between the elements corresponds to the edge of the directed graph. According to the description of the plate-making process, the order of the points and lines is connected by the directed edges, that is, the garment plate-making process knowledge directed graph model is obtained.

\subsection{Model Construction}

Different clothing cartographer differ in the methods and habits used to describe their platemaking process statements, which are personal experiences. However, from the substance of the sentence description, there are common parts. Analyze and summarize the plate-making description sentences in Section 2.3, and obtain the following general plate-making description sentences. In each sentence, brackets can be filled with symbols or names that identify corresponding points and lines.

Sentence A: Take (point/line) as the starting point ( ) to ( ) ( ) fixed point ( )

Sentence B: Over ( ) ( ) (horizontal / vertical) ( ) ( ) at point ( ) 
Sentence C: Take ( ) as the starting point (horizontal/vertical) to ( ) ( )

Sentence D: Take ( ) as a starting point ( ) ( ) parallel ( )

Sentence E: Take ( ) as a starting point and ( ) take a fixed point ( )

Sentence F: Take ( ) (midpoint/three equal point) ( )

Sentence G: draw a circle with ( ) as the center and ( ) as the radius ( )

Sentence H: Connection ( ) ( ) completed ( )

The process model of the plate-making structure of the suit collar is constructed as shown in Fig. 4.

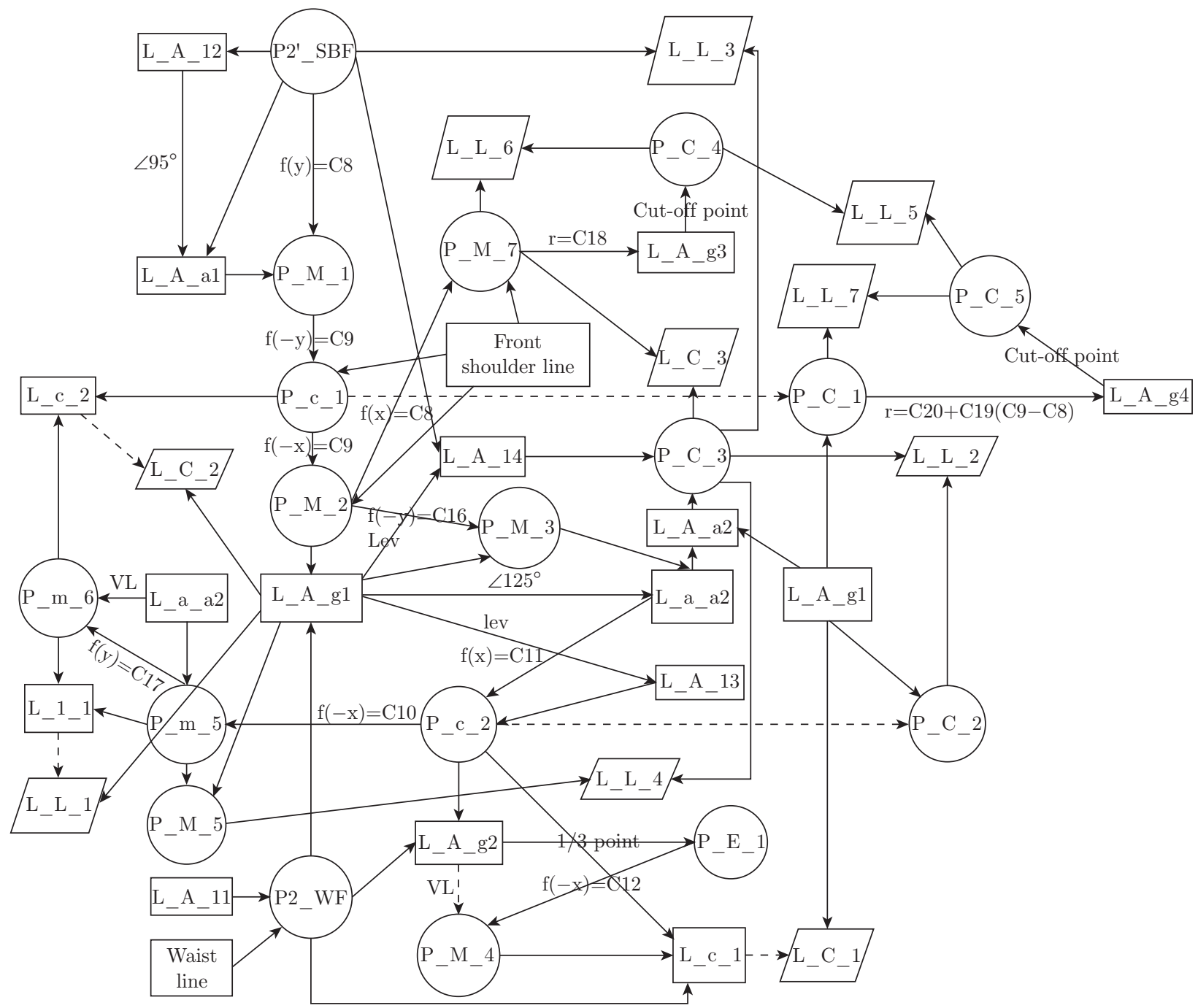

Fig. 4: Suit collar plate-making process knowledge model

It can be seen from Fig. 4 that the points, lines and directions in the plate-making process are the three basic elements in the pattern forming process. The data (numerical, size formula, plate making formula), direction, and parallel, vertical and other relationships are the bridges that connect the three major elements. Therefore, the knowledge in the plate-making process includes not only the data, parallel and vertical relationships between points and lines, but also the order of the plate-making process. 


\subsection{Model Analysis}

The expression content of the garment patterning process knowledge directed graph model is a visual description of the knowledge of the garment plate-making process, and its expression is more detailed and intuitive. It not only includes all the points, lines and data (values, size formulas, plate making formulas), parallel, vertical and other explicit design knowledge applied in the garment plate making process. It also includes the orderly implicit design knowledge between points and line elements in the plate making process.

For the knowledge-driven smart clothing CAD systems, the garment plate-making process knowledge directed graph model can effectively carry out layout design and modification. Take the suit collar process knowledge model constructed in Section 3.2 as an example, corresponding to Fig. 2, P_c_2 is the tip point of the suit collar. If the design of the point is adjusted according to the customer's requirements, then according to the suit collar plate-making process knowledge, the directed graph model can quickly and easily find the mapping elements directly affected by the change of the point including P_m_5, P_C_2, L_A_g2. From these three elements, all other change elements P_M_5, L_l_1, P_E_1, etc. can be found. Finally, according to the order of the arrows, a complete inspection of the model is completed, and it can be seen that the adjustment of the plate-making element point P_c_2 can affect the suit-type structure lines L_C_1, L_C_2, L_L_2, as shown in Fig. 5. The design of the pattern can be flexibly designed and modified according to the order of the orientation and the numerical and geometric constraints between the plate-making elements.

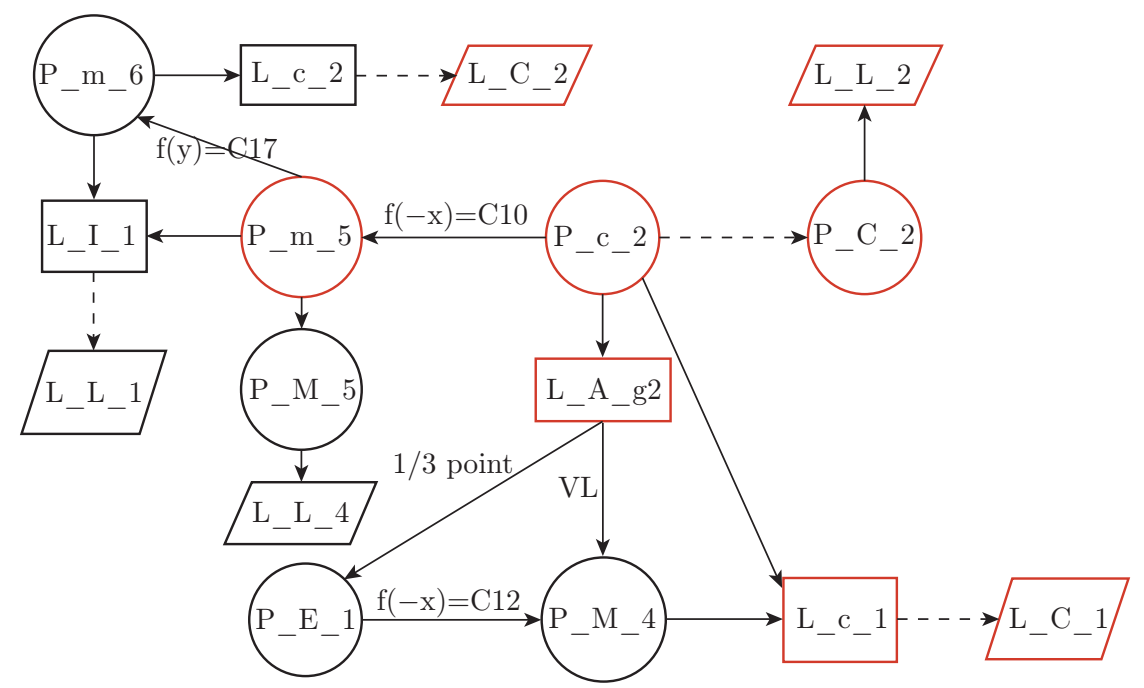

Fig. 5: Pointing order of P_c_2

\section{Conclusion}

Based on the development of intelligent clothing plate-making software, this paper analyses and obtains the process knowledge of the suit collar plate-making method, as well as presents the directed graph model of knowledge of the plate-making process according to the basic theory of graph theory. This paper further analyzes the constructed model by examples, highlights the 
integrity and analyzability of the model for the knowledge representation of the garment platemaking process, and provides a theoretical method for the automation of garment pattern design knowledge for the knowledge-driven garment intelligent CAD systems.

\section{Acknowledgements}

The name of the project is "Research on Constructing a Garment Pattern Design Model for Garment Intelligent Design" (Project No. J201606), which is supported by the China Textile Industry Federation and Textile Light Technology Education Foundation.

\section{References}

[1] Lu H, Liu GL, Dai HQ. Influence of Overturned Collar Width on Gradient Data of Lapel Collar. Text Res J: 2006; 8; 45-48.

[2] Feng J, Zhang HZ. Parametric Design of Rolled Collar Pattern. Journal of Tianjin Polytechnic University: 2006; 5; 79-81.

[3] Zhang YY, Sun ZK. Digital Plate-making Technology of Men's Suit Collar. Wool Textile Journal: 2017; 10; 52-55.

[4] Jiang LJ. Research on Collar 3D Construction Digital Technology Based on Neck Characteristic. Donghua University: 2010; 32-36.

[5] Feng F, Liu JF. The Design Principle and Application of Dynamic Clothing Structure. Journal of ZJFIT: $2011 ; 4 ; 25-28$.

[6] Hu CP. The Digitized Research of Suit Intelligent Generated Pattern Design System. Beijing Institute of Fashion: 2010; 77-83.

[7] Jiang ZH. Construct Component Library of Men's Suit and Establish Parametric Model of Suit Template. Xi'an Polytechnic University: 2014; 43-47.

[8] Zhao MM. A Study on the Method of Knowledge Acquisition and Expression of Uniform Design Based on Case Reasoning. Xi'an Polytechnic University: 2014; 55-57.

[9] Duan J. Research on Relational Model for Pattern of Menswear based on Digitizing 3D Human Model. Xi'an Polytechnic University: 2017; 65-67.

[10] Zhou WC. Construction of Intelligent Clothing Pattern Model Based on Domain Ontology. Xi'an Polytechnic University: 2016; 23-27.

[11] Liu RP. Women's Clothing Pattern Design Principles and Applications. 2008, Beijing: China Textile Press: 340-366.

[12] Liu RP. Men's Clothing Pattern Design Principles and Applications. 2008, Beijing: China Textile Press: 132-136.

[13] Zhang P. Parallel Transformation of Serial-program Under Multi-core Platform. University of Electronic Science and Technology of China: 2015; 11-15.

[14] Qian TD. Modern Graph Theory. 1987, Beijing: Higher Education Press: 1-3. 